\title{
¿ES LA INVERSIÓN PÚBLICA EN EDUCACIÓN UNA POLÍTICA REDISTRIBUTIVA? \\ Un análisis de la composición del gasto por nivel de enseñanza
}

\author{
IS PUBLIC INVESTMENT ON EDUCATION A REDISTRIBUTIVE POLICY? \\ An Analysis on the Composition of Expenditure by Educational Level
}

\author{
Dulce Manzano \\ Fundación Juan March. Madrid \\ Dmanzano@march.es \\ LeIRE SALAZAR \\ UNED \\ Lsalazar@poli.uned.es
}

\section{RESUMEN}

En este trabajo se discute y analiza la dimensión redistributiva del gasto público en educación mediante un examen comparado de una amplia muestra de países desarrollados entre 1960 y 1996. Los estudios existentes que examinan los determinantes de la financiación pública de la educación suelen suponer que ésta tiene un carácter progresivo. En nuestro análisis se cuestiona esta visión y, partiendo del supuesto de que es la composición del gasto entre los distintos niveles de enseñanza, y no el nivel de gasto total, lo que realmente tiene consecuencias distributivas, se aporta evidencia sobre el peso de la ideología del partido en el gobierno y la posición económica del votante mediano en la configuración de esta política.

\section{Palabras Clave Adicionales}

Capital humano, Desigualdad, Gasto público, Partidos políticos, Redistribución.

\section{Abstract}

In this article, we examine the redistributive nature of public educational spending through a cross-country analysis from 1960 to 1996. Existing studies on the determinants of the extent of public funding of education tend to give for granted its progressive nature. We question this view and, assuming that the redistributive nature of public education is conditional on how expenditure is allocated between the different levels of schooling, we provide evidence on the role of the ideological nature of the party in government and of the economic position of the median voter on the configuration of this policy.

\section{Additional KeYWORdS}

Human Capital, Inequality, Political Parties, Public Spending, Redistribution. 


\section{INTRODUCCIÓN}

Como consecuencia del creciente proceso de internacionalización de las economías nacionales que ha tenido lugar a partir de los años ochenta, los gobiernos se han enfrentado a mayores limitaciones para redistribuir mediante políticas estándar por el lado de la demanda. En la literatura se ha considerado, no obstante, que los políticos gozan de un cierto margen de maniobra para implementar políticas redistributivas por el lado de la oferta (Boix, 1997). Típicamente, la provisión de educación pública es una política clave de este tipo de programas en la medida en que se considera que su impacto redistributivo no presenta costes muy elevados para la eficiencia de la economía. Empíricamente, se ha utilizado el gasto público en educación como un indicador de dicha política. Sin embargo, no todo el gasto en educación supone una redistribución progresiva, dado que ésta depende de cómo se distribuya el presupuesto educativo entre los distintos niveles de enseñanza.

Este trabajo parte del supuesto de que es la composición y no el nivel total de gasto educativo la dimensión que realmente tiene implicaciones distributivas. Este supuesto encuentra apoyo amplio en la literatura sociológica sobre desigualdad de oportunidades educativas y en diversos análisis empíricos sobre la distribución de los beneficios de la política educativa entre los distintos grupos de ingresos. Nuestro objetivo es analizar, por un lado, en qué medida distintos tipos de partidos -con preferencias ideológicas y/o bases de apoyo diferentes- optan por combinaciones alternativas de gasto en los niveles de primaria y terciaria. Por otro lado, se tienen en cuenta las constricciones electorales a las que deben hacer frente los partidos mediante una exploración de la influencia de la posición política del votante mediano (atribuida a partir de su ubicación relativa en la distribución de los ingresos) que, teóricamente, cambia con el grado de desigualdad económica.

El artículo está estructurado de la siguiente manera. En primer lugar, discutimos los argumentos teóricos aportados por la literatura relevante y exponemos nuestras hipótesis. El siguiente apartado está dedicado a la contrastación empírica de nuestras hipótesis describiendo, en primer lugar, los datos y métodos utilizados en la estimación de los modelos de regresión y, en segundo lugar, presentando los resultados empíricos de nuestras estimaciones. En la última sección concluimos con un breve resumen de los principales hallazgos obtenidos de estos análisis.

\section{GAStO EDUCATIVO, PARTIDOS Y DESIGUALDAD}

En la literatura fragmentada sobre los determinantes del gasto educativo destacan una serie de análisis que plantean la existencia de un conflicto de intereses en torno a la provisión pública de educación. Estos estudios comparten la visión de que la financiación pública en educación es una política de naturaleza redistributiva que 
acarrea una transferencia de recursos de las clases más favorecidas hacia los grupos menos aventajados de la sociedad. En los trabajos en los que se formalizan las consecuencias distributivas de esta política (Ansell, 2006; Saint-Paul y Verdier, 1993), se suele suponer que la educación pública es un bien uniforme que se distribuye de manera igualitaria y que se financia con un esquema fiscal progresivo: un impuesto proporcional al ingreso. En consecuencia, la inversión gubernamental en educación beneficia a las clases más pobres en detrimento de los individuos en los tramos altos del ingreso -sobre los que recae la mayor parte del peso de la financiación. Dado este esquema redistributivo, es razonable pensar que las condiciones económicas relativas de los individuos determinarán sus preferencias sobre el grado ideal de financiación pública y, en concreto, que los grupos con mayores ingresos desearán limitar el nivel de gasto educativo.

Las hipótesis sustantivas que proponen estos estudios sobre los determinantes del nivel de implicación estatal en el gasto en educación podrían dividirse en dos líneas argumentativas. Por una parte, se encuentra una hipótesis en torno a la ideología del partido gubernamental, formulada en la literatura centrada en países desarrollados como los de la OCDE. Según esta hipótesis, los partidos políticos sostienen distintas preferencias por el gasto educativo debido a que representan a distintos grupos de ingreso que constituyen sus bases electorales de apoyo. Así, los partidos de izquierda o socialdemócratas se preocuparían, fundamentalmente, por el bienestar de las clases menos acomodadas de la sociedad, mientras que los partidos conservadores responderían, idealmente, a los intereses de los individuos con mayores recursos. En consecuencia, y dada la naturaleza redistributiva de la provisión pública de educación descrita anteriormente, cabe esperar, de acuerdo con esta hipótesis, que los gobiernos de izquierda aumenten la financiación en la enseñanza pública y que sus homólogos de derecha tengan interés en contener (o incluso reducir) el gasto educativo para limitar las contribuciones fiscales de las clases con mayores recursos (Castles, 1989; Boix, 1996; 1997; Ansell, 2006; Busemeyer, 2007).

De acuerdo con el argumento de Boix (1996; 1997), quizás el autor más influyente en el debate sobre partidos y educación, la creciente internacionalización de la economía que ha ocurrido a partir de la década de los ochenta ha incrementado las limitaciones a las que se enfrentan los gobiernos para redistribuir mediante políticas tradicionales por el lado de la demanda. Sin embargo, los gobiernos han logrado mantener un cierto margen de maniobra para implementar políticas redistributivas por el lado de la oferta. Para Boix, una política intensiva de formación de capital humano a través de un mayor gasto público en educación se dirige a incrementar las cualificaciones de la fuerza de trabajo en general y de los trabajadores menos cualificados en particular. Este tipo de políticas beneficia, principalmente, a los segmentos menos cualificados (más pobres) de la sociedad, fomentando la igualdad. Por lo tanto, la promoción del gasto público en formación constituye una de las principales estrategias económicas con las que los partidos socialdemócratas cuentan para fomentar la igualdad en contextos económicos que hacen difícil el uso de políticas keynesianas. 
Una segunda hipótesis que se propone en los análisis formales sobre la economía política de la educación (por ejemplo, Saint-Paul y Verdier, 1993) establece un vínculo causal entre la desigualdad y la provisión pública de educación. Además de la ideología del gobierno, es lógico pensar que las demandas de los votantes más decisivos en las elecciones influyen también en la configuración de las políticas. Los partidos políticos tratarán de satisfacer a estos votantes por razones puramente electorales. Estas constricciones electorales, formalizadas en los modelos clásicos de competición partidista a través de la posición del votante mediano, inducen a los partidos a ajustar sus políticas hacia las preferencias de este votante para mejorar sus probabilidades de éxito electoral.

Cuando la política sigue un esquema estándar de redistribución, como el de la educación pública en el modelo de Saint-Paul y Verdier (1993)'1 las preferencias del votante (o ingreso) mediano van a estar determinadas por su distancia económica respecto a la media. En este contexto, el votante mediano siempre tenderá a favorecer cierto nivel redistributivo dado que su ubicación en la distribución de la renta (que se corresponde con el ingreso mediano) se encuentra por debajo de la media. Es más, sus preferencias redistributivas serán mayores a medida que aumente la desigualdad, ya que esto se traduce en un incremento de la diferencia de ingreso entre la media y la mediana. Así, la hipótesis que se deriva tanto del trabajo de Saint-Paul y Verdier como de otros estudios acerca de la educación pública que se basan en este tipo de modelos es que la desigualdad económica tendrá un impacto positivo en el gasto público en educación.

Este trabajo parte, a diferencia de los anteriores estudios, de que la educación pública no tiene por qué estar asociada de manera inequívoca con un programa de redistribución unidireccional a favor de los grupos menos acomodados de la sociedad. Apoyándonos en la abundante literatura en el campo de la estratificación social que confirma la existencia de desigualdad de oportunidades educativas, suponemos que las consecuencias redistributivas del gasto público van a depender en gran medida de cómo se asigne el gasto total entre los distintos niveles de enseñanza. Entre las líneas de investigación más fértiles de este campo en las últimas décadas se encuentra el estudio de la relación entre orígenes sociales y logro educativo. Uno de los principales hallazgos indiscutibles de la evidencia empírica acumulada hasta el momento a este respecto consiste en la existencia de desigualdad de oportunidades educativas: la proporción de individuos que acceden a los distintos niveles educativos varía en función de la posición socioeconómica de sus familias de origen.

Existe bastante consenso sobre el aumento en el logro educativo medio de las distintas cohortes nacidas en el último siglo. Los niveles básicos de escolarización primaria y, en algunos casos, educación secundaria obligatoria- se han universalizado

\footnotetext{
${ }^{1}$ Es decir, una política financiada por un impuesto proporcional al ingreso cuyos beneficios se distribuyen igualitariamente entre toda la población.
} 
como consecuencia de la adopción de reformas educativas destinadas a ampliar el número de años de educación obligatoria. La expansión de los niveles más altos, en concreto universitarios, ha tenido menor alcance. Pero el hecho de que los estudiantes continúen en el sistema educativo en proporciones mayores a lo largo del tiempo es ciertamente compatible con la persistencia de un acceso diferencial en cada nivel para estudiantes con distintos orígenes socioeconómicos. Pese a que estos cambios agregados se deberían haber traducido en una reducción de la desigualdad educativa, empíricamente no podemos apoyar esta conclusión de manera inequívoca en todos los países. A principios de la década de los noventa, los principales estudios comparados entre países (Shavit y Blossfeld, 1993) establecieron cierto consenso de que, a pesar de que proporciones mayores en sucesivas cohortes accedían a los niveles educativos no obligatorios, en la mayor parte de países desarrollados la desigualdad de oportunidades educativas permaneció inalterada. Estudios más recientes -tanto de un solo país como comparados- apuntan, sin embargo, a que durante el siglo XX ha tenido lugar cierta reducción de este tipo de desigualdad en un número considerable de países europeos (Jonsson et al., 1996; Shavit y Westerbeek, 1998; Breen, 2004; Vallet, 2004), aunque se ha demostrado que el efecto del origen socioeconómico ha permanecido relativamente constante en otros países como Estados Unidos (Mare, 1993).

En la medida en que el acceso a primaria esté garantizado de manera universal y financiado por un sistema fiscal progresivo, se puede concluir que un mayor gasto en primaria implica un beneficio neto para las clases más desfavorecidas. En el caso de la formación en secundaria, no está tan clara la identificación de qué grupos se benefician en mayor medida de la inversión en este nivel. Además de que su grado de obligatoriedad entre los distintos países varía, el peso de las trayectorias académica versus profesional según el origen social difiere en gran medida entre los distintos sistemas educativos. En muchos países, sería recomendable distinguir además entre educación secundaria obligatoria y post-obligatoria para captar la inclusión progresiva de la primera en el total de años obligatorios de estudio. Sin embargo, no existen datos apropiados de gasto público por separado para estos dos sub-niveles, de modo que en este trabajo se considera toda la educación secundaria como un único nivel.

A diferencia de lo que ocurre en educación secundaria y, sobre todo, en primaria, el hecho de que la enseñanza universitaria no sea obligatoria genera una fuerte desigualdad de clase en el acceso a este nivel, incluso en los países desarrollados de la OCDE. Siempre que exista cierto grado de desigualdad de oportunidades en el acceso a educación terciaria, su naturaleza redistributiva sería cuestionable -y, sin lugar a dudas, menor que en primaria- incluso si este tipo de formación fuera completamente financiado de manera pública con impuestos proporcionales a los ingresos de los contribuyentes. Los individuos o las familias que pertenecen a orígenes socioeconómicos más privilegiados, ubicados en la parte más alta de la distribución de la renta, podrán extraer, en media, ganancias netas de la educación terciaria y, en consecuencia, un nivel de gasto público más alto en terciaria no necesariamente implicaría una redistribución mayor de ricos a pobres. 
En efecto, Hills (2004) descubre, usando datos de ingresos y gasto social para el Reino Unido, que los individuos situados en el quintil más rico de la distribución reciben dos tercios más del valor monetario estimado de la educación terciaria que el grupo de ingreso más pobre, un hallazgo que le lleva a concluir que la inversión en educación universitaria resulta ser una política que favorece relativamente a la clase más rica (Hills, 2004: 191). De acuerdo con su análisis empírico, la financiación de la universidad pública es la única medida a favor de la clase más aventajada en comparación con otras políticas sociales como el gasto sanitario, en vivienda pública o atención a dependientes, cuyos beneficiarios están concentrados en los tramos más bajos de la renta. Además, Hills muestra que la composición del presupuesto educativo tiene un fuerte impacto distributivo en la medida en que el gasto en primaria tiende a estar dirigido a los individuos relativamente más pobres, mientras que la educación en terciaria tiende a generar beneficios netos a los individuos o grupos más privilegiados de la sociedad. Para el caso de España, San Segundo (2001) también muestra evidencia sobre la desigualdad en el acceso a la universidad según el nivel educativo alcanzado por la familia de origen.

En este trabajo, cuando examinamos la composición del gasto educativo nos centramos exclusivamente en la inversión pública en los niveles más alto y bajo del sistema educativo -primaria y terciaria-, al suponer que representan los escenarios redistributivos más extremos en términos de quiénes son sus principales beneficiarios. Partimos, por lo tanto, del supuesto de que una configuración del gasto que dé prioridad al nivel de primaria sobre el de terciaria tendrá mayores consecuencias redistributivas a favor de las clases más pobres.

En la medida en que los grupos de renta que se benefician varían con el nivel educativo, argumentamos que los efectos de los factores típicamente asociados con el total de gasto educativo propuestos en la literatura podrían ser compatibles con efectos que operan de forma opuesta en cada nivel de formación y que, en consecuencia, examinar el total de gasto podría no ser la manera más apropiada de captar el conflicto de intereses que tiene lugar en la asignación de la inversión pública en educación. Al poder un mismo nivel de gasto total estar asociado con tipos de composición que favorecen a distintas clases sociales, nuestra hipótesis primera es que deberíamos observar que variables como la ideología del gobierno o la desigualdad no tienen por qué tener un efecto sistemático sobre el gasto público en educación.

Nuestra segunda hipótesis relaciona la ideología del partido gubernamental con los beneficios percibidos por los distintos grupos de renta de la configuración del gasto entre terciaria $(T)$ y primaria $(P)$. Los partidos socialdemócratas, cuyas bases electorales se localizan fundamentalmente entre los grupos de ingresos más bajos y, en principio, orientados en mayor medida por objetivos de igualdad, optarán por una política de gasto educativo centrada en primaria; en cambio, los partidos conservadores, atendiendo a los intereses de sus bases electorales, tradicionalmente pertenecientes a los tramos más altos de la distribución de ingresos, tenderán a favorecer políticas de financiación que concentran en mayor medida los recursos hacia la enseñanza superior. 
Nuestra tercera hipótesis vincula la desigualdad con la asignación del gasto en terciaria y primaria. Puesto que los partidos políticos han de responder a las preferencias del votante mediano para conseguir mayorías electorales, el posicionamiento de este votante respecto a la política influirá también en la determinación de los niveles relativos de gasto en terciaria y primaria. En la medida en que el votante mediano se beneficie en menor grado de la educación superior conforme aumenta la desigualdad, como consecuencia del empeoramiento de sus condiciones económicas, es de esperar que la desigualdad tenga un efecto negativo sobre el peso relativo de la educación universitaria en la composición del gasto.

\section{ANÁLISIS EMPÍRICO}

\section{Datos y métodos}

En esta sección explicamos brevemente las fuentes de los distintos tipos de datos en los que basamos nuestros análisis empíricos -datos que, como explicamos más adelante, proceden, por una parte, de una recopilación propia a partir de los anuarios sobre educación disponibles para varios años y, por otra parte, de otras fuentes secundarias-, describimos la definición y operacionalización de nuestras variables, y brevemente aludimos, por último, a los métodos utilizados en los modelos de estimación de este trabajo.

Restringimos nuestra muestra inicial de casos a los 30 países de la OCDE más los países europeos no pertenecientes a esta organización -en total, 53 países, incluyendo Estados con fronteras territoriales que han cambiado a lo largo del tiempo. Por otro lado, nuestra ventana temporal de partida comprende los años entre 1960 y 1996. Evidentemente, no para todas las variables utilizadas en el análisis las series disponen de información sobre todos los países y años, de modo que el número de observaciones (países por años) efectivo sobre la que se realizan las estimaciones del análisis empírico disminuye considerablemente a medida que éstas incluyen especificaciones más completas.

Las dos variables dependientes - gasto total en educación y porcentaje de gasto en los diferentes niveles de formación- han sido construidas tomando toda la información disponible en la serie completa de anuarios estadísticos publicados por la UNESCO entre 1977 y 1998 (UNESCO, varios años)². La variable de gasto total en educación se define

\footnotetext{
2 En concreto, se han extraído datos de los anuarios de 1977, 1980-1984, 1986-1987, 1991-1994 y 19961998. Dado que los anuarios incluyen información retrospectiva sobre las variables, en algunas ocasiones en las que existe solapamiento en los años para los que se incluye información pero en los que los valores difieren, hemos optado por considerar el valor del anuario más reciente como el más preciso, al suponer que éste recoge los posibles ajustes que la propia organización haya realizado en las series.
} 
como el porcentaje del Producto Nacional Bruto (PNB) que se invierte en educación, y tiene en cuenta tanto los gastos ordinarios como los gastos en capital. Los anuarios consultados ofrecen, además, información detallada sobre el gasto (solamente ordinario, en este caso) en los distintos niveles de formación como porcentaje del total de gasto en educación, si bien estas series son más incompletas que las que corresponden al gasto educativo total, por lo que al utilizar la información sobre niveles reducimos en cierta medida el tamaño muestral. Hemos construido nuestra segunda variable dependiente como la razón entre el gasto en educación superior y el gasto en primaria. Mientras la definición del nivel terciario tiende a ser sistemática en las series entre países y dentro de ellos a lo largo del tiempo, en no pocos casos los niveles inferiores a secundaria no se encuentran suficientemente desagregados, por lo que la educación pre-escolar y primaria han sido tomadas conjuntamente en todos los análisis para evitar la pérdida de un importante número de casos. La razón de gasto entre educación terciaria y primaria (T/P) es pues una medida relativa de la inversión que los gobiernos realizan en un nivel (educación universitaria) en comparación con otro (nivel inferior a secundaria). Adopta un valor igual a la unidad cuando no existe diferencia en el gasto en ambos niveles, valores por encima de uno cuando la magnitud en el numerador (en este caso, el gasto en universidad) es superior a la magnitud en el denominador (gasto en enseñanza primaria) $^{3}$ y valores por debajo de la unidad siempre que el denominador sea más alto. Al ser nuestro interés analizar las reasignaciones del presupuesto que tienen lugar exclusivamente entre estos dos niveles, el gasto en secundaria (como porcentaje del total del presupuesto) se incluye como variable de control para no atribuir erróneamente variaciones en la razón de gasto entre terciaria y primaria que en realidad se deban a cambios en el gasto en secundaria.

Para medir la ideología del partido en el gobierno utilizamos una variable que distingue si el partido es de derecha, de izquierda o de centro, tomada de la versión actualizada en 2004 de los datos Database of Political Institutions (DPI, 2001) de Beck et al. (2001). En la práctica, para simplificar los análisis empíricos y la interpretación de los resultados, se ha excluido la categoría "centro" (que solamente representa un $7 \%$ del total de observaciones), de modo que en todo el trabajo nuestro indicador IZQUIERDA es una variable dicotómica que adopta valor cero para gobiernos de ideología conservadora y valor uno en el caso de gobiernos con ideología socialdemócrata.

Utilizamos el coeficiente de Gini como medida agregada de desigualdad, que adopta valor cero cuando la distribución de los ingresos es totalmente igualitaria y valor cien cuando ésta presenta el mayor grado posible de desigualdad -valores mayores indican, por lo tanto, distribuciones más desiguales. El hecho de que esta medida esté acotada por estos dos valores y que no sea sensible al número de observaciones disponibles en cada muestra, hace que sea una de las medidas sintéticas de desigualdad más

\footnotetext{
${ }^{3}$ Estrictamente, gasto en enseñanza pre-escolar y primaria, aunque por razones prácticas nos referimos en el trabajo a este nivel como "primaria".
} 
frecuentemente utilizadas para comparar niveles a lo largo del tiempo y/o entre países. En la práctica, en nuestra muestra no encontramos valores extremos del Gini, que tiene un rango entre 20 y 50. Los datos que utilizamos proceden de las series ofrecidas por el Luxembourg Income Study (LIS). LIS es un proyecto de investigación a gran escala y a largo plazo que, desde 1983, recopila datos nacionales de 30 países en el nivel individual y del hogar, procedentes de diversas fuentes y sobre diferentes conceptos de ingreso, y los armoniza para optimizar su comparación en el tiempo y entre países. En las LIS Key Figures 4 , se proporcionan datos sobre niveles de desigualdad (coeficientes de Gini) calculados a partir de las distintas bases de datos individuales armonizadas para todos los países y años disponibles ${ }^{5}$. Hemos realizado una interpolación de estos índices de Gini para completar las series temporales; aún así, debemos señalar que, dada la escasez de datos sobre desigualdad económica de calidad, especialmente si uno desea remontarse considerablemente en el tiempo, como es nuestro caso, la introducción de esta variable reduce significativamente nuestra muestra de países/años. Los valores resultantes de la interpolación son los que utilizamos como nuestra principal medida de desigualdad a lo largo del trabajo $0^{6}$.

El resto de variables de control que se incluyen en las distintas estimaciones se explican en detalle en la sección de resultados empíricos. Para todas ellas, discutimos las razones teóricas que recomiendan su incorporación en cada uno de los análisis, los efectos que esperamos que tengan sobre las variables dependientes y la procedencia de los datos. En el apéndice, se incluye una tabla (Tabla 3) con los principales estadísticos descriptivos de todas las variables utilizadas en los análisis de regresión.

Para poner a prueba nuestras hipótesis, estimamos modelos de regresión lineal con Mínimos Cuadrados Ordinarios con diferentes especificaciones para cada una de nuestras dos variables dependientes -el gasto total en educación (como porcentaje del PNB) y la razón de gasto en terciaria en comparación con primaria (T/P). A pesar de que los datos consisten en series temporales para un número relativamente elevado de países, en la mayor parte de las especificaciones nuestro interés sustantivo está más orientado a la comparación entre contextos con configuraciones diferentes de nuestras variables que a los patrones temporales de éstas. En este sentido, no explotamos de manera sistemática la naturaleza dinámica de los datos y, en consecuencia, la

\footnotetext{
${ }^{4}$ Véase http://www.lisproject.org/keyfigures.htm para más información sobre estos datos.

${ }^{5} \mathrm{El}$ concepto concreto de ingreso que se utiliza es renta disponible equivalente (definida como la renta disponible en el hogar ajustada por el tamaño del mismo) excluyendo los valores iguales a cero.

${ }^{6}$ Una medida alternativa (o, más bien, complementaria) de igualdad que refleja de forma más precisa la posición económica del votante mediano (o ingreso mediano) es el porcentaje de los ingresos totales que está en manos del tercer quintil de la distribución de ingresos. La base de datos habitualmente utilizada para realizar comparaciones internacionales es Deininger y Squire (1996) y su actualización por el Instituto Mundial para la Investigación del Desarrollo Económico de las Naciones Unidas (UNU-WIDER, 2005). Sin embargo, el limitado número de observaciones disponibles hace que se reduzca considerablemente la muestra sobre la que se basan nuestros análisis. Por ello hemos optado por el uso del coeficiente de Gini.
} 
mayor parte de nuestras estimaciones no aplican métodos específicos para analizar datos de panel. La notable persistencia en el tiempo de las dos principales variables independientes -ideología y desigualdad- también hace recomendable la adopción de una perspectiva transversal. Aun así, para descartar posibles sesgos derivados de la no consideración del aspecto temporal, hemos puesto a prueba la consistencia de nuestros principales resultados teniendo en cuenta las características no observadas propias de cada país -estimando, por lo tanto, especificaciones con efectos fijos en los que se analizan cambios a lo largo del tiempo dentro de los países.

\section{Resultados del análisis empírico}

En esta sección, presentamos los hallazgos empíricos más destacados de este trabajo. Por una parte, ilustramos la relaciones entre nuestras variables dependientes y nuestras variables independientes de interés -la desigualdad y la ideología de los partidos en el gobierno- mediante un examen descriptivo simple de los datos. Este análisis nos permite detectar el grado de variabilidad de los fenómenos que queremos explicar y, de una manera muy preliminar, sus pautas de asociación con los factores explicativos sugeridos por nuestras hipótesis. Por otra parte, mostramos y discutimos los resultados de varios modelos de regresión lineal que, de forma sistemática, ponen a prueba las hipótesis de trabajo.

Para comprobar la validez de la primera hipótesis, que cuestiona la visión convencional de que la provisión pública de educación es una política redistributiva que promueve la igualdad, examinamos en qué medida la ideología del gobierno y la desigualdad inicial están relacionadas con el nivel de gasto educativo. A modo de evidencia descriptiva de nuestro argumento, en el Gráfico 1 se representa la correlación entre desigualdad y gasto total en educación para todos los países y años que forman parte de nuestra muestra y que, además, disponen de información sobre estas variables. El gráfico muestra claramente que no existe una relación positiva entre el nivel de desigualdad y el gasto educativo. De hecho, de existir algún tipo de asociación, los datos parecen apuntar a que ésta es negativa. La posición extrema de México en ambos ejes (el grupo de observaciones con un valor del coeficiente de Gini próximo a 50) podría estar agudizando esta pauta; sin embargo, incluso si elimináramos este país de la muestra, parece evidente que la relación en ningún caso se haría positiva, en contra de las predicciones de los estudios anteriores. Además, se puede descartar que la ausencia de una relación positiva se deba a la influencia de la ideología del gobierno. En el gráfico se diferencian las observaciones según la ideología del ejecutivo y no es posible concluir que la desigualdad incremente el gasto total para ninguno de los tipos de partidos.

En cuanto a la tesis del efecto de los partidos, si los gobiernos socialdemócratas realmente utilizaran esta política como estrategia de redistribución, deberíamos observar que, para cada valor de la desigualdad, su nivel de gasto educativo se encuentra por encima del de los partidos conservadores. Como se puede apreciar en el gráfico, los partidos de izquierda no tienden, en general, a presentar unas tasas de gasto mayores que los de derecha, excepto para el tramo más bajo de la desigualdad. 
Gráfico 1.

Relación entre gasto público total en educación y desigualdad

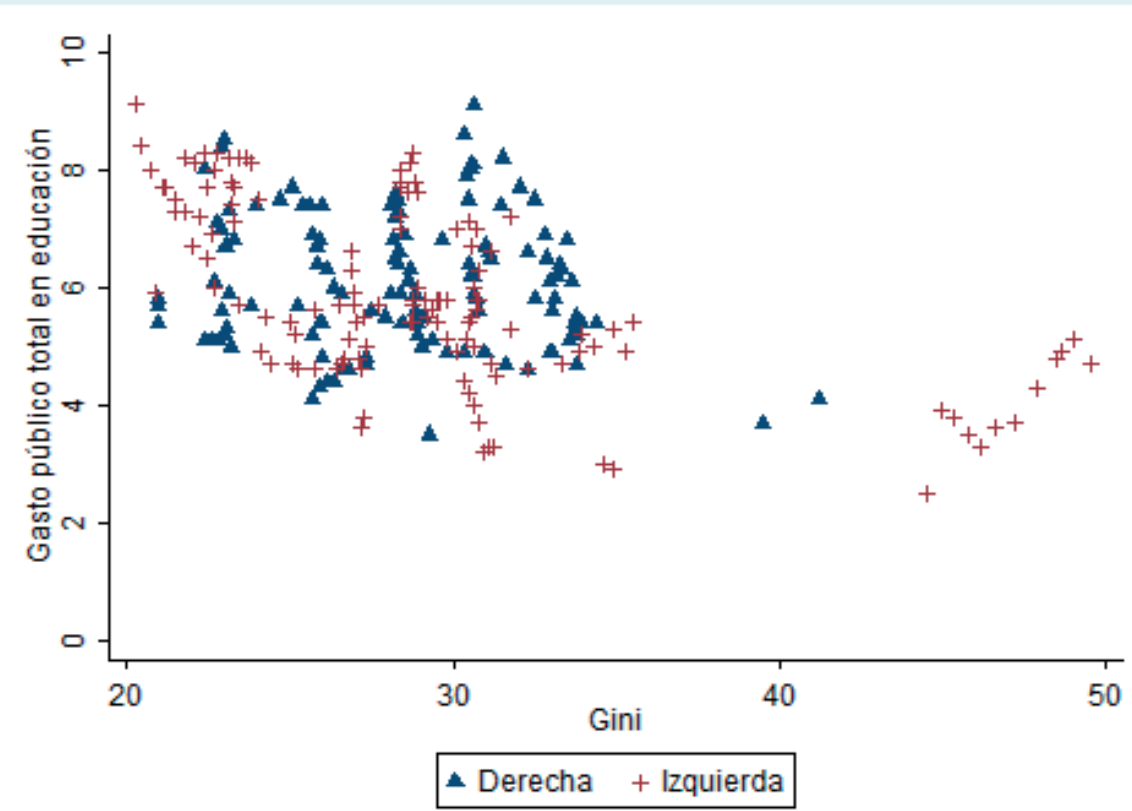

Para comprobar de manera más rigurosa nuestra primera hipótesis, hemos estimado varias especificaciones relativas a un modelo de regresión lineal cuyos resultados se presentan en la Tabla 1. En la primera especificación solamente se incluyen los factores explicativos de interés -ideología y desigualdad. De acuerdo con los coeficientes de las variables, el efecto del color del partido en el gobierno es negativo aunque no significativo; este resultado, en línea con nuestra tesis de que el gasto total en educación no necesariamente debe constituir una política redistributiva progresiva, indica que el presupuesto destinado a educación es estadísticamente independiente de la ideología del partido gubernamental. El coeficiente de regresión asociado a la variable GINI también parece apoyar nuestra hipótesis en la medida en que no es positivo y es, además, estadísticamente significativo. Lejos de aumentar la financiación en educación, incrementos en la desigualdad están asociados con una menor tasa de gasto público.

En la especificación 2 contrastamos en qué medida estos hallazgos son robustos a la inclusión de los controles clásicos que se suelen manejar en la literatura. La variable INGRESO, que sirve como proxy del desarrollo económico, se refiere al PIB real per cápita en dólares constantes (año base 1996) obtenido de la versión 6.1 de la base de datos Penn World Table (Heston, Summers y Aten, 2002). Aparte de la razón obvia 
Tabla 1.

Gasto público total en educación (\% del PNB)

\begin{tabular}{|c|c|c|c|c|}
\hline & (1) & (2) & (3) & (4) \\
\hline IZQUIERDA & $\begin{array}{l}-0.160 \\
(0.149)\end{array}$ & $\begin{array}{l}-0.092 \\
(0.152)\end{array}$ & $\begin{array}{l}-0.198 \\
(0.162)\end{array}$ & $\begin{array}{l}0.097 \\
(0.139)\end{array}$ \\
\hline GINI & $\begin{array}{l}-0.110 \\
(0.012)^{\star \star *}\end{array}$ & $\begin{array}{l}-0.158 \\
(0.018)^{\star \star *}\end{array}$ & $\begin{array}{l}-0.162 \\
(0.021)^{\star \star *}\end{array}$ & $\begin{array}{l}-0.017 \\
(0.032)\end{array}$ \\
\hline INGRESO & & $\begin{array}{l}0.118 \\
(0.022)^{\star \star \star}\end{array}$ & $\begin{array}{l}0.115 \\
(0.022)^{\star \star \star}\end{array}$ & $\begin{array}{l}-0.250 \\
(0.040)^{\star \star *}\end{array}$ \\
\hline POBL $<14$ & & $\begin{array}{l}0.132 \\
(0.021)^{\star * *}\end{array}$ & $\begin{array}{l}0.136 \\
(0.020)^{\star * \star}\end{array}$ & $\begin{array}{l}-0.091 \\
(0.056)\end{array}$ \\
\hline RURAL & & $\begin{array}{l}0.005 \\
(0.007)\end{array}$ & $\begin{array}{l}0.006 \\
(0.007)\end{array}$ & $\begin{array}{l}-0.169 \\
(0.107)\end{array}$ \\
\hline APERTURA COMERCIAL & & $\begin{array}{l}0.003 \\
(0.003)\end{array}$ & $\begin{array}{l}0.002 \\
(0.003)\end{array}$ & $\begin{array}{l}0.008 \\
(0.008)\end{array}$ \\
\hline DÉCADAS (Dummies) & & & sí & sí \\
\hline PAÍSES (Dummies) & & & & sí \\
\hline Constante & $\begin{array}{l}9.236 \\
(0.365)^{\star * \star}\end{array}$ & $\begin{array}{l}5.456 \\
(0.909)^{\star * *}\end{array}$ & $\begin{array}{l}6.246 \\
(0.941)^{\star \star \star}\end{array}$ & $\begin{array}{l}16.289 \\
(2.651)^{\star \star \star}\end{array}$ \\
\hline Observaciones & 253 & 238 & 238 & 238 \\
\hline $\begin{array}{l}\text { R-cuadrado } \\
\text { R-cuadrado (intra-grupos) } \\
\mathrm{N} \text { de países }\end{array}$ & 0.22 & 0.44 & 0.47 & $\begin{array}{l}0.83 \\
0.23 \\
24\end{array}$ \\
\hline
\end{tabular}

Nota: La variable INGRESO está expresada en miles. Errores típicos robustos entre paréntesis. "significativo al $10 \%$; ** significativo al $5 \%$; ${ }^{* * *}$ significativo al $1 \%$.

de que cuanto más desarrollado es un país mayor será, en principio, su capacidad de ampliar los servicios públicos de educación, existen otros posibles mecanismos que afectan tanto a la demanda educativa como a los incentivos del gobierno a aumentar la formación de capital humano. Es razonable pensar que en sociedades más desarrolladas (sujetas a procesos crecientes de terciarización de la economía), los individuos estén más dispuestos a invertir en su formación como medio de mejora de sus posiciones en el mercado de trabajo en la medida en que el vínculo entre educación y oportunidades laborales se refuerza (Shavit y Blossfeld, 1993). Además, otra explicación del impacto positivo del desarrollo económico es que la productividad de las cualificaciones de la fuerza de trabajo se eleva en etapas posteriores del proceso de industrialización. Según Galor y Moav (2004), la importancia relativa del capital físico y humano como motores del crecimiento económico cambia con la industrialización creciente de la economía. En 
las etapas iniciales del desarrollo, los beneficios de la formación del capital humano son más bajos que los del capital físico, de manera que el papel productivo del primero en el crecimiento económico es limitado. Sin embargo, a medida que aumenta la acumulación de capital, el trabajo cualificado adquiere una importancia mayor para sostener las tasas de beneficio y el crecimiento económico. Así pues, se podría pensar que los gobiernos, con independencia de sus preferencias ideológicas, tenderán aumentar la provisión pública de educación con el desarrollo económico.

La proporción de la población menor de 14 años (población en edad escolar, $\mathrm{POBL}<14$ ) es otro factor relevante que debe ser incluido en el modelo para controlar por la composición demográfica de los países -que afecta a los resultados educativos y a los esfuerzos que los gobiernos deben hacer para conseguirlos. Dado un mismo objetivo respecto a la cantidad o calidad de la educación (por ejemplo, tasas de escolarización en primaria y secundaria), cuanto mayor es la fracción de población joven de una sociedad, a priori, mayores recursos se precisan para alcanzarlo. Según este argumento, el efecto de este indicador demográfico sobre nuestra variable dependiente debería ser positivo. La fuente de estos datos es World Development Indicators 2000 recogidos por el Banco Mundial (2000).

La variable RURAL, que también procede de los datos de World Development Indicators 2000, indica la proporción de población rural como porcentaje del total de población. Existen razones sustantivas para esperar tanto un efecto positivo como uno negativo del peso del sector primario de la economía sobre el gasto en educación. Por una parte, la provisión de educación en áreas rurales es más costosa debido a que, al estar su población menos concentrada, se requiere una inversión fija mayor que en áreas urbanas ${ }^{7}$. Para lograr unos mismos resultados educativos (por ejemplo, de escolarización), en sociedades con una fracción mayor de población rural serán precisas asignaciones superiores del presupuesto público que en sociedades con un grado de urbanización más alto. Este mecanismo apunta, por lo tanto, hacia una relación positiva entre ambas variables. Por otra parte, sin embargo, es de esperar que la población rural tenga menores incentivos a adquirir educación debido a que la demanda de trabajadores altamente cualificados es más baja en los mercados laborales agrícolas. La existencia de este segundo mecanismo, con un efecto negativo sobre la financiación de la educación, dejaría, en nuestro modelo, abierta la posibilidad de una relación neta tanto positiva como negativa dependiendo del grado en que ambas influencias se compensen.

El último control de la especificación 2 es el total de importaciones y exportaciones como porcentaje del PIB que tomamos como indicador del grado de apertura comercial de un país. Estos datos están recogidos por el Banco Mundial en su base de datos World Development Indicators 2000. Cabe esperar que los países, a partir de un cierto nivel de desarrollo, dependan de una fuerza de trabajo más cualificada para mantener sus tasas de crecimiento económico conforme se intensifica su exposición

\footnotetext{
${ }^{7}$ Para un argumento similar pero aplicado al tamaño de las naciones, véase Alesina y Spolaore (1997).
} 
a la competencia internacional. Puesto que la ventaja competitiva de estas naciones se basa, fundamentalmente, en productos o servicios que requieren mano de obra con altas dotaciones de capital humano, es de esperar que las inversiones en educación crezcan cuanto más abiertas y sujetas estén sus economías a las dinámicas de los mercados internacionales.

Incluyendo estos cuatro controles en la regresión básica (especificación 2 de la Tabla 1), se observa que los resultados referentes a la desigualdad y a la ideología del partido gubernamental apenas se ven modificados. El coeficiente de la variable IZQUIERDA mantiene su signo negativo y sigue sin ser significativo. Al igual que en la anterior especificación, la desigualdad tiende a reducir significativamente la inversión pública en educación, en oposición al argumento convencional de que un votante mediano empobrecido demandará un mayor nivel de gasto total educativo porque las ganancias que obtiene del programa redistributivo que implica la educación pública son mayores. Según las predicciones de este trabajo, el desarrollo económico y la proporción de población en edad escolar afectan positivamente a la variable dependiente. El grado de apertura comercial parece no ser un factor relevante que contribuya a explicar la variación del gasto educativo. Por último, la fracción de población rural en la sociedad no produce ningún cambio estadísticamente significativo en el porcentaje del PNB que se dedica a educación pública. Es probable que la ausencia de significatividad de este control sea el resultado de que los efectos (positivo y negativo), descritos anteriormente, se compensan.

En la siguiente especificación, columna cuarta en la Tabla 1, hemos añadido indicadores dicotómicos de las décadas para anular la posible tendencia temporal de la variable dependiente. La introducción de estas dummies temporales nos permite controlar la parte de la covarianza entre el gasto público y los factores explicativos del modelo que se deba a la existencia de una tendencia a lo largo del tiempo en las variables. De esta forma, evitamos que las relaciones estimadas sean espurias en lo que respecta a la dimensión dinámica de los datos. Como se puede observar en la tabla, todos los resultados sustantivos derivados del modelo se mantienen inalterados, pese a la inclusión de estos controles temporales.

Ante la posibilidad de que estas estimaciones se deban a la omisión de características no observadas específicas de los países, en la especificación 4 replicamos el análisis incluyendo efectos fijos de los grupos (países). Centrándonos únicamente en la variación a lo largo del tiempo dentro de los países -consecuencia de la estimación con efectos fijos-, apreciamos que algunos de los coeficientes de la regresión cambian. La modificación más importante consiste en que la desigualdad pierde su capacidad explicativa $^{8}$ y la ideología pasa a ser positiva, aunque se mantiene no significativa. En

\footnotetext{
${ }^{8}$ La pérdida de significatividad de esta variable puede deberse a que la desigualdad es bastante persistente en el tiempo en la mayoría de los países que componen nuestra muestra, de manera que existe escasa variación en los niveles de desigualdad dentro de cada grupo.
} 
cualquier caso, esta evidencia continúa siendo consistente con nuestra primera hipótesis de que el gasto público total en educación no necesariamente implica, por sí mismo, una redistribución progresiva. Respecto a las variables de control, se pueden destacar dos cambios principales: primero, incrementos a lo largo del tiempo en el ingreso per cápita tienden a reducir significativamente los valores de la variable dependiente; segundo, la proporción de población menor de 14 años deja de ser un factor relevante de la explicación.

El examen de nuestras R-cuadrado sugiere que el total de variación explicada por nuestras sucesivas especificaciones es considerable en todas las especificaciones. Por otra parte, y como cabe esperar, esta variación explicada aumenta en gran medida en la última especificación, lo que indica que las características no observables de los países aportan un notable peso a la explicación.

En nuestro argumento, no cuestionamos ni la relación negativa entre desigualdad y posición económica del ingreso o votante mediano, ni que sus preferencias redistributivas se intensifiquen a medida que crece la desigualdad. Utilizando la razón entre gasto en terciaria y primaria como indicador de la composición del presupuesto educativo, mostramos evidencia sobre el efecto de las posiciones partidistas y la desigualdad sobre la distribución de dicho presupuesto entre estos dos niveles de enseñanza. El resto de esta sección está dedicada a la comprobación de las hipótesis 2 y 3.

Gráfico 2.

Relación entre distribución del gasto público en educación

T/P (terciaria/primaria) y desigualdad.

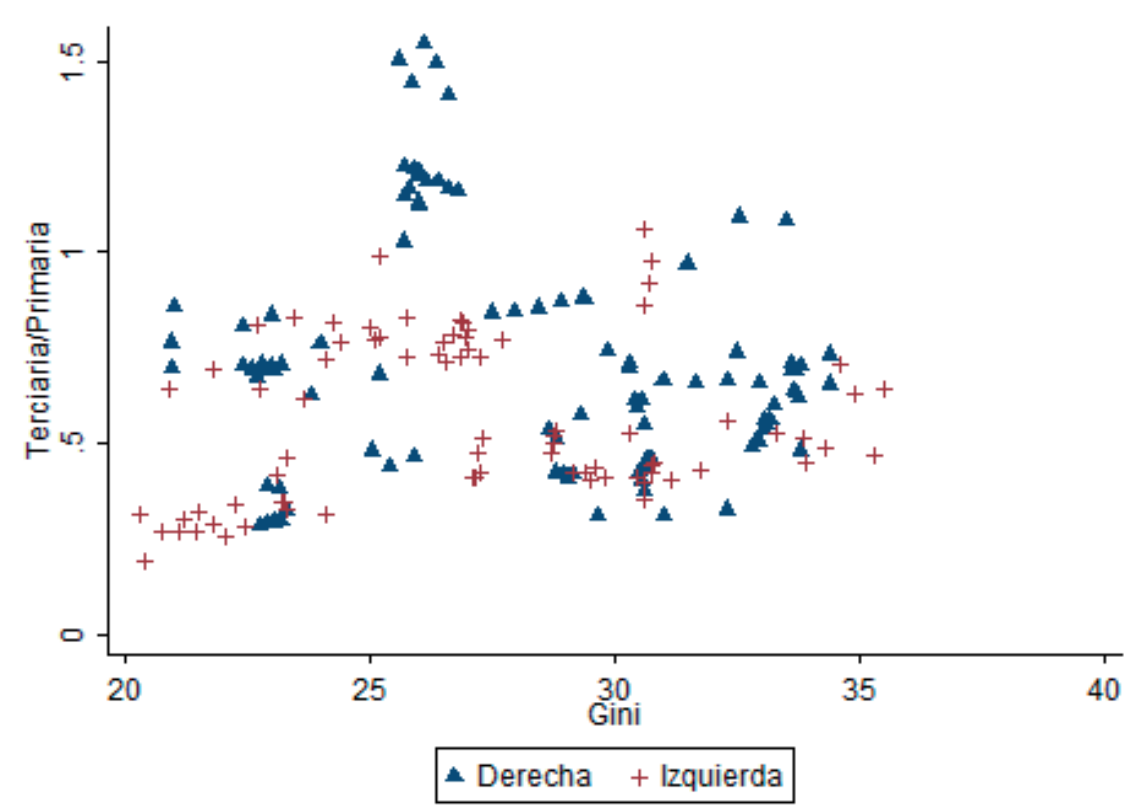

RIS, VOL. 67, № 3, SEPTIEMBRE-DICIEMBRE, 655-679, 2009. ISSN: 0034-9712 
El Gráfico 2 representa de manera exploratoria la relación existente entre el Gini y la razón del gasto entre terciaria y primaria en nuestra muestra, distinguiendo según la ideología del partido en el poder. A simple vista, aunque los datos no muestran un patrón de asociación demasiado claro tomando todas las observaciones, si consideramos las submuestras para cada tipo de partido parece sugerirse que la desigualdad tiene un efecto negativo más acentuado en gobiernos conservadores que en gobiernos socialdemócratas. De hecho, si ajustáramos una recta de regresión para los casos de los últimos, ésta sería prácticamente plana. La interpretación de esta pauta estaría indicando que los partidos de izquierda, a diferencia de los de derecha, no parecen reasignar las partidas del gasto educativo entre terciaria y primaria como consecuencia de cambios en la distribución de la renta. A diferencia de lo que se apreciaba en el gráfico anterior, en este caso es más claro que cuando los partidos conservadores están en el poder, la razón T/P tiende a adoptar valores superiores que con gobiernos socialdemócratas, como apuntábamos en las hipótesis. Además, los datos indican que esta diferencia se mantiene para casi todos los valores de la desigualdad.

Con el fin de evaluar el impacto neto de la desigualdad y de la ideología de los partidos, estimamos una serie de regresiones lineales que descuentan la influencia que pudieran tener otras variables relevantes sobre la distribución del gasto educativo. Los resultados de éstas se presentan en la Tabla 2. La segunda columna de la tabla constituye la especificación básica sin controles en la que únicamente se introducen nuestras principales variables de interés, además del gasto en secundaria que, por las razones antes explicadas, nos permite aislar la reasignación del gasto que tiene lugar sólo entre los dos niveles que nos interesan: terciaria y primaria. De acuerdo con los resultados de esta especificación, los coeficientes de las variables sustantivas, IZQUIERDA y GINI, tienen el signo esperado y son estadísticamente significativos.

En consonancia con la hipótesis segunda, los gobiernos de izquierda presentan, en media, niveles menores en la razón de gasto T/P que los de derecha. Parece confirmarse, pues, que las preferencias partidistas importan en la determinación de cómo se distribuye el presupuesto educativo entre los distintos niveles de enseñanza. Además, se sostiene empíricamente nuestra hipótesis de que los grupos políticos socialdemócratas optan por una configuración de la inversión pública en educación menos orientada hacia terciaria que sus homólogos conservadores, puesto que un descenso relativo del gasto en terciaria (a favor de primaria) favorece a los sectores menos aventajados de la sociedad. Esta influencia negativa de la ideología de izquierda sobre la variable dependiente es robusta en las distintas regresiones que se muestran en la tabla. La comparación de esta pauta sistemática con la ausencia de efecto de la ideología que detectábamos en la explicación del gasto público total refuerza nuestra crítica a la utilización del gasto total como indicador del impacto redistributivo de la educación.

Nuestra tercera hipótesis también se confirma a la vista de las estimaciones de esta primera especificación básica. El coeficiente asociado a la variable GINI indica que, efectivamente, el grado de desigualdad disminuye el peso relativo de terciaria a favor 
del gasto en educación primaria9. Si los partidos en el gobierno, por razones electorales, se ven forzados a moderar sus preferencias políticas teniendo en cuenta las demandas del votante mediano, nuestro argumento es que cuanto mayor es el nivel de desigualdad (es decir, cuanto más pobre es el votante mediano), la razón T/P debería adoptar valores más bajos, como parece confirmarse en la regresión estimada en la especificación 1.

Tabla 2.

Distribución del gasto público en educación T/P (terciaria/primaria)

\begin{tabular}{|c|c|c|c|c|c|}
\hline & (1) & (2) & (3) & (4) & (5) \\
\hline \multirow[t]{2}{*}{ IZQUIERDA } & -0.152 & -0.078 & -0.400 & -0.540 & -0.418 \\
\hline & $(0.037)^{\star \star \star}$ & $(0.036)^{\star *}$ & $(0.236)^{*}$ & $(0.273)^{\star \star}$ & $(0.192)^{\star *}$ \\
\hline \multirow[t]{2}{*}{ GINI } & -0.010 & -0.004 & -0.014 & -0.010 & -0.036 \\
\hline & $(0.004)^{\star *}$ & $(0.005)$ & $(0.006)^{\star *}$ & $(0.006)^{*}$ & $(0.008)^{\star * *}$ \\
\hline \multirow[t]{2}{*}{ GASTO SECUNDARIA } & 0.011 & 0.006 & 0.010 & 0.005 & 0.003 \\
\hline & $(0.002)^{\star \star \star}$ & $(0.003)^{\star *}$ & $(0.002)^{\star * *}$ & $(0.003)$ & $(0.003)$ \\
\hline \multirow[t]{2}{*}{ GINI*IZQUIERDA } & & & 0.009 & 0.017 & 0.013 \\
\hline & & & $(0.008)$ & $(0.010)^{*}$ & $(0.007)^{\star}$ \\
\hline \multirow[t]{2}{*}{ INGRESO } & & 0.013 & & 0.014 & 0.030 \\
\hline & & $(0.005)^{\star *}$ & & $(0.005)^{* *}$ & $(0.011)^{\star \star *}$ \\
\hline \multirow[t]{2}{*}{ APERTURA COMERCIAL } & & 0.004 & & 0.004 & 0.001 \\
\hline & & $(0.001)^{\star \star *}$ & & $(0.001)^{\star \star *}$ & $(0.002)$ \\
\hline \multirow[t]{2}{*}{ LOG(POBL) } & & 0.105 & & 0.105 & -0.808 \\
\hline & & $(0.033)^{\star \star \star}$ & & $(0.031)^{\star \star *}$ & $(0.290)^{\star \star *}$ \\
\hline \multirow[t]{2}{*}{ RÉGIMEN } & & 0.177 & & 0.181 & -0.026 \\
\hline & & $(0.084)^{\star \star}$ & & $(0.081)^{\star *}$ & $(0.050)$ \\
\hline \multirow[t]{2}{*}{ GASTO TOTAL EDUC } & & 0.025 & & 0.031 & 0.079 \\
\hline & & $(0.024)$ & & $(0.023)$ & $(0.023)^{\star * *}$ \\
\hline DÉCADAS (Dummies) & & sí & & sí & sí \\
\hline PAÍSES (Dummies) & & & & & sí \\
\hline \multirow[t]{2}{*}{ Constante } & 0.605 & -1.822 & 0.731 & -1.691 & 13.935 \\
\hline & $(0.123)^{\star \star \star}$ & $(0.655)^{\star \star *}$ & $(0.199)^{\star * \star}$ & $(0.627)^{\star \star \star}$ & $(4.939)^{\star \star *}$ \\
\hline $\begin{array}{l}\text { Observaciones } \\
\text { R-cuadrado } \\
\text { R-cuadrado (intra-grupos) } \\
\mathrm{N} \text { de países }\end{array}$ & $\begin{array}{l}179 \\
0.23\end{array}$ & $\begin{array}{l}164 \\
0.31\end{array}$ & $\begin{array}{l}179 \\
0.23\end{array}$ & $\begin{array}{l}164 \\
0.32\end{array}$ & $\begin{array}{l}164 \\
0.89 \\
0.37 \\
20 \\
\end{array}$ \\
\hline
\end{tabular}

Nota: La variable INGRESO está expresada en miles. Errores típicos robustos entre paréntesis. *significativo al $10 \% ;{ }^{* *}$ significativo al $5 \% ;{ }^{* * *}$ significativo al $1 \%$.

\footnotetext{
${ }^{9}$ Recuérdese que el gasto en secundaria permanece constante.
} 
Con la segunda especificación de la Tabla 2 se demuestra hasta qué punto estas relaciones se mantienen al introducir otros factores que potencialmente están asociados con la composición del gasto. Para descartar que la influencia de las variables independientes sobre la magnitud de la inversión en terciaria vis-à-vis primaria opere a través del nivel de gasto público total, incluimos esta variable en el análisis.

Consideramos de nuevo el desarrollo económico (INGRESO) porque es posible esperar que los cambios estructurales entre sectores económicos que éste implica se asocien a una mayor demanda de trabajo cualificado, que podría causar una reasignación del presupuesto educativo a favor de la enseñanza universitaria. Nótese que el mecanismo relacionado con la disponibilidad de recursos que se aplicaba en el análisis anterior, no opera en la configuración del gasto entre terciaria y primaria una vez controlado el monto total de recursos destinados a la educación pública.

En cuanto a la variable de la apertura comercial de la economía, retomamos la argumentación que ofrecíamos para justificar su inclusión en la explicación del gasto educativo total. En la medida en que los países relativamente desarrollados requieren

Gráfico 3.

Efecto de la desigualdad en T/P (Terciaria/Primaria) según la ideología del gobierno

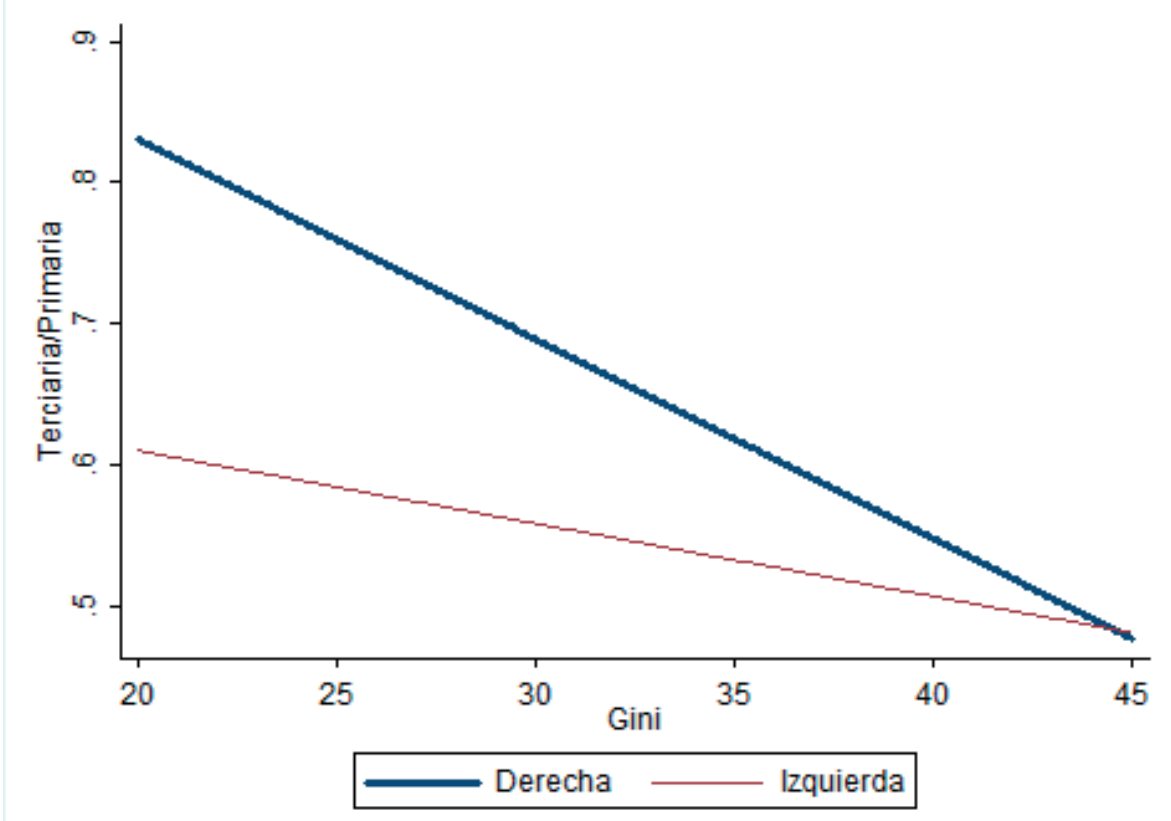


una fuerza de trabajo altamente cualificada para mantener su competitividad en los mercados internacionales, conforme aumente su integración económica será más probable que intensifiquen sus esfuerzos de gasto en niveles superiores de formación.

Cuando examinábamos el gasto total en educación incorporábamos una medida de la composición demográfica que consistía en la proporción de población en edad escolar. En el caso de la política de gasto en T/P, el efecto de esta variable vendría en cierta forma recogido por el gasto en secundaria. Como alternativa, optamos por el tamaño de la población como la característica demográfica más relevante. Es razonable pensar que, en países con un tamaño de la población reducido, la inversión adicional en educación universitaria sea, en términos relativos, más costosa que la inversión en niveles inferiores. Si la cobertura de la educación primaria es universal, se requerirá un mínimo de dotación en este nivel de formación en todos los núcleos de población -independientemente de su tamaño-, mientras que cabe esperar que el número total de centros de enseñanza universitaria en estos contextos demográficos sea limitado. Así pues, el efecto esperado del tamaño de la población es que aumente la razón de gasto entre terciaria y primaria. Esta variable, LOG(POBL), procedente de la base de datos World Development Indicators 2000 (Banco Mundial, 2000), se incluye en nuestro modelo en forma logarítmica.

Por último, en países dictatoriales con ideología de izquierda (por ejemplo, en nuestra muestra, algunas observaciones correspondientes a Polonia), la fuerte presencia de la educación pre-escolar -que permite la plena incorporación de las mujeres al mercado laboral- podría reducir la razón entre el gasto en terciaria y el gasto en primaria ${ }^{10}$. Para evitar que estos casos distorsionen los resultados estadísticos, se ha incorporado un indicador dicotómico referido al régimen político (valor 0 para democracia y 1 para dictadura) que descuenta el efecto de este fenómeno. Los datos para esta variable proceden de Przeworski et al. (2000).

De acuerdo con los coeficientes de la especificación 2, todos los controles introducidos se comportan de la manera esperada y sus efectos son estadísticamente significativos. En sociedades más desarrolladas y con una mayor apertura comercial, los valores de la razón T/P tienden a ser más altos. Los países con un tamaño de la población mayor y con gobiernos dictatoriales parecen, según nuestros datos, tener una asignación presupuestaria relativa en educación terciaria más elevada. Respecto a nuestras hipótesis sustantivas, como ya hemos comentado, el indicador de la ideología de los partidos es significativo y su coeficiente tiene el signo esperado. La desigualdad provoca un descenso en la variable dependiente tal y como predice nuestra hipótesis, aunque en esta especificación su coeficiente no resulta significativo.

En la discusión del Gráfico 2 se señalaba de manera preliminar la posible existencia de una asociación diferencial entre la desigualdad y la distribución del gasto dependiendo

\footnotetext{
${ }^{10}$ Recordamos que el gasto en primaria incluye también, como describimos en la sección de Datos, la educación pre-escolar.
} 
del color del partido en el gobierno. Con el fin de evaluar la incidencia de estos patrones con métodos estadísticos de regresión, las especificaciones 3 y 4 de la Tabla 2 reproducen los dos últimos análisis introduciendo la interacción entre la desigualdad y la dummy de la ideología. Este término interactivo mide el cambio del impacto de la desigualdad en contextos políticos de izquierda en comparación con los de derecha -adviértase que la categoría de referencia del modelo corresponde a los partidos de derecha. Además, esto nos permite determinar hasta qué punto las políticas que implementan distintos tipos de partidos están condicionadas por el grado de desigualdad -es decir, por la ubicación económica y, en consecuencia, por la política ideal del votante mediano. Atendiendo a los coeficientes de la especificación sin controles, se observa que el correspondiente al GINI es negativo y significativo, y que el parámetro de la interacción tiene signo positivo pero no es estadísticamente significativo. Para ilustrar los mecanismos sustantivos derivados de este modelo interactivo, el Gráfico 3 muestra cómo cambian los valores predichos de la regresión ante variaciones de la desigualdad, distinguiendo por la ideología del partido gubernamental ${ }^{11}$.

Se puede confirmar que, como ya apuntaba el Gráfico 2, existen pautas de relación distintas en función de la ideología del gobierno. En escenarios de mayoría conservadora, la desigualdad influye negativamente y de manera significativa en el gasto de terciaria sobre primaria. Aunque la desigualdad actúa en la misma dirección en el caso de gobiernos socialdemócratas, la magnitud de tal influencia es menos acentuada; la prueba de significatividad del impacto de la desigualdad en la izquierda (que es la suma de los coeficientes de la variable GINI y del término de interacción) indica, de hecho, que este efecto no es significativo. Sustantivamente, estos resultados sugieren que los partidos de derecha suelen ajustar la política de composición del gasto educativo a los cambios en las preferencias del votante mediano, a diferencia de los partidos socialdemócratas. Una razón plausible que puede explicar por qué estos últimos no responden en la misma medida es que parten de niveles iniciales preferidos de T/P inferiores $y$, por tanto, el ajuste que deben realizar al empeorar la situación económica del votante mediano es más moderado.

El Gráfico 4 pone a prueba si las distancias partidistas respecto a la configuración del gasto se ven alteradas en los distintos niveles de desigualdad ${ }^{12}$. El eje vertical representa las diferencias predichas entre gobiernos de distinto corte ideológico y el eje horizontal representa distintos niveles de desigualdad económica (Gini). La línea continua más gruesa mide el efecto de la ideología, es decir, las diferencias predichas entre los partidos de izquierda y derecha para cada valor del coeficiente Gini. Las dos

\footnotetext{
${ }^{11}$ En esta simulación, la variable GASTO SECUNDARIA se ha mantenido constante en su valor medio para la muestra utilizada en la regresión.

${ }^{12} \mathrm{Al}$ igual que en la simulación anterior, en este caso también se mantiene constante la variable GASTO SECUNDARIA en su valor medio. Los detalles técnicos sobre el procedimiento utilizado para la construcción de este gráfico se encuentran explicados en Brambor et al. (2006).
} 
Gráfico 4.

Efecto de la ideología del gobierno en T/P (Terciaria/Primaria) para distintos niveles de desigualdad.

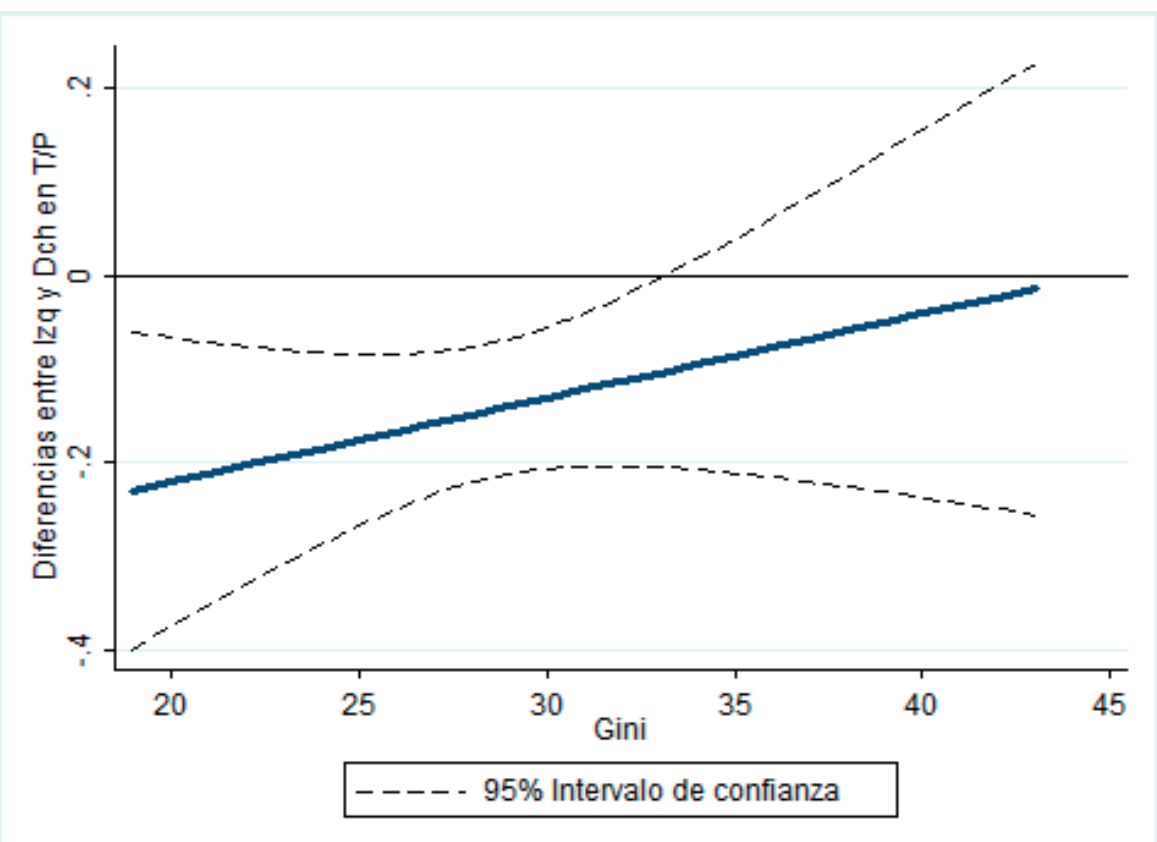

líneas discontinuas marcan el intervalo de confianza que determina la significatividad de la distancia entre partidos. La línea horizontal en el valor cero marca la ausencia de diferencias partidistas en cuanto a la política de interés. Por tanto, un valor negativo en la línea continua más gruesa significa que los partidos socialdemócratas implementan, en promedio, una razón de gasto T/P más baja. Para los valores de la desigualdad (Gini) en los que el intervalo de confianza incluye la línea horizontal fija en el valor cero, se puede afirmar que el efecto de la ideología no es significativo.

La primera cuestión que se debe destacar es que los valores de la divergencia partidista están sistemáticamente por debajo de cero; esto implica que el peso del gasto en terciaria (relativo al de primaria) suele ser superior en países con gobiernos conservadores, confirmando nuestra segunda hipótesis. No obstante, las diferencias disminuyen con la desigualdad económica e incluso pierden su significatividad a partir de valores intermedios del coeficiente de Gini. Esta pauta es consecuencia de la evidencia anterior de que las respuestas de los gobiernos de izquierda, ante incrementos de la desigualdad, son más moderadas.

Los dos últimos análisis de la Tabla 2 se refieren el modelo interactivo básico incorporando todos los controles que hemos discutido (especificación 4) y, además, las 
características no observadas específicas de los países (especificación 5). En cuanto a los factores de control, la novedad más destacable consiste en que la medida del tamaño de la población pasa a tener un coeficiente negativo y significativo cuando estimamos el modelo usando únicamente la variación dentro de los grupos. Este cambio de efecto podría deberse a que, al centrarnos en la variación en el tiempo dentro de cada de país, la variable ya no se refiere al tamaño de la población sino a aumentos en éste producidos, muy probablemente, por entradas importantes de contingentes de inmigrantes. La inversión adicional que deben realizar los gobiernos para acomodar las necesidades educativas de estos nuevos inmigrantes estará más enfocada a los niveles inferiores de enseñanza que a la educación universitaria. Respecto a nuestras hipótesis sustantivas, las conclusiones a las que llegamos en el modelo interactivo básico -acerca de las distancias partidistas y del impacto diferencial de la desigualdad según la ideología del partido gubernamental- se mantienen en ambas especificaciones, con la excepción de que, en el caso de la regresión con efectos fijos, la asociación negativa entre el Gini y la razón T/P se hace significativa en contextos de mayoría socialdemócrata.

\section{Conclusiones}

Existen influyentes teorías económicas que consideran al capital humano como una fuente crucial del crecimiento económico. La presencia de externalidades positivas atribuidas, en esta perspectiva, a la inversión en educación, ha alimentado la idea de que la intervención estatal en formación genera beneficios netos globales para toda la economía. Un creciente número de estudios reconoce la existencia de una heterogeneidad de preferencias en torno a la política educativa en función de las posiciones económicas de los individuos. Se señala que la política educativa comporta una distribución de recursos entre las clases sociales o grupos de ingreso y, por lo tanto, puede generar un conflicto de intereses en torno al nivel de transferencias y financiación educativas.

En este trabajo se comparte esta visión; sin embargo, se cuestiona la concepción común a esta literatura de que las consecuencias distributivas de la educación pública operan, de manera inequívoca, a favor de los individuos con menores rentas. Los hallazgos presentados en esta sección corroboran, en términos generales, la validez de nuestras tres hipótesis. El hecho de que la ideología de los partidos y la desigualdad no estén sistemáticamente relacionadas con la cantidad total de recursos públicos asignados a la educación en la dirección apuntada por la literatura, apoya nuestra crítica a estos estudios: que esta política no necesariamente tiene que acarrear una redistribución a favor de los sectores más pobres de la sociedad. Nuestra propuesta teórica consiste en que la naturaleza redistributiva de la política educativa depende de cómo se distribuyan los recursos entre los distintos niveles de enseñanza. Una política que dé prioridad a la financiación de terciaria a expensas de la de primaria implica una mayor concentración de los beneficios en torno a las clases más aventajadas. Si esto es así, nuestra segunda 
hipótesis sugiere que este tipo de medida educativa será más frecuente, en promedio, en países de mayoría conservadora que en aquellos con partidos socialdemócratas en el poder, como efectivamente demuestran los análisis. Por último, proponemos que los partidos adaptan sus preferencias a cambios en las condiciones económicas del electorado y, en concreto, de los votantes que ocupan posiciones decisivas para formar mayorías electorales (en un modelo básico de competición electoral, el votante mediano). La evidencia de que incrementos en la desigualdad -que empeoran la situación económica relativa del votante mediano- tienden a reducir la razón de gasto T/P encaja con nuestra tercera hipótesis, si bien queda abierta para investigaciones futuras una exploración detalla de las razones por las que el impacto de la desigualdad para los gobiernos de izquierda no son tan robustos.

\section{RefERENCIAS BibLIOGRÁFICAS}

Alesina, A. y E. Spolaore. 1997. "On the Number and Size of Nations". Quarterly Journal of Economics 112: 1027-1056.

Ansell, B. 2006. From the Ballot to the Blackboard. The Redistributive Political Economy of Education. Departamento de Gobierno. Universidad de Harvard: tesis doctoral.

Banco Mundial. 2000. World Development Indicators 2000. Washington D.C. Banco Mundial: CD-ROM.

Beck, T., G. Clarke, A. Ggroff, P. Keeper y P. Walsh. 2004. "New Tools in Comparative Political Economy: The Database of Political Institutions". World Bank Economic Review 15: 165-176.

Boix, C. 1996. Partidos políticos, crecimiento e igualdad. Estrategias económicas conservadoras y socialdemócratas en la economía mundial. Madrid: Alianza Universidad.

Boix, C. 1997. "Political Parties and the Supply Side of the Economy: The Provision of Physical and Human Capital in Advanced Economies, 1960-90". American Journal of Political Research 41: 814-845.

Brambor, T., W. Clark y M. Golder. 2006. "Understanding Interaction Models: Improving Empirical Analyses". Political Analysis 14: 63-82.

Breen, R. (coord.) 2004. Social Mobility in Europe. Oxford: Oxford University Press.

Busemeyer, M. R. 2007. "Determinants of Public Education Spending in 21 OECD Democracies, 1980-2001". Journal of European Social Policy, 14: 582-610.

Castles, F. 1989. "Explaining Public Education Expenditure in OECD Nations". European Journal of Political Research 17: 431-448.

Deininger, K. y L. Squire. 1996. "Measuring Inequality: A New Data-Base". World Bank Economic Review, 10: $565-591$.

Galor, O. y O. Moav. 2004. "From Physical to Human Capital Accumulation: Inequality and the Process of Development". Review of Economic Studies 71: 1001-1026. 
Heston, A., R. Summers y B. Aten. 2002. Penn World Table Version 6.1, Center for International Comparisons at the University of Pennsylvania (CICUP).

Hills, J. 2004. Inequality and the State. Oxford: Oxford University Press.

Jonson, J. O., C. Mills y W. Müller. 1996. "A Half Century of Increasing Educational Openness? Social Class, Gender and Educational Attainment in Sweden, Germany and Britain". En R. Erikson y J. O. Jonsson (coord.), Can Education Be Equalized? The Swedish Case in Comparative Perspective, pp. 183-206. Boulder: Westview Press.

Luxembourg Income Study (LIS) Key Figures, descargado de la página web http://www.lisproject.org/ keyfigures.htm en agosto de 2007.

Mare, R.D. 1993. "Educational Stratification on Observed and Unobserved Components of Family Background". En Y. Shavit y H.P. Blossfeld (coord.), Persistent Inequality: Changing Educational Attainment in Thirteen Countries: 351-76. Boulder: Westview Press.

Prezeworski, A., M. Alvarez, J. Cheibub y F. Limongi. 2000. Democracy and Development. Nueva York: Cambridge University Press.

Saint-Paul, G. y T. Verdier. 1993. "Education, Democracy and Growth". Journal of Development Economics 42: 399-407.

San Segundo, M.J. 2001. Economía de la Educación. Madrid: Síntesis.

Shavit, Y. y H.P. Blossfeld. 1993. Persistent Inequality: Changing Educational Attainment in Thirteen Countries, (coord.), Boulder: Westview Press.

Shavit, Y. y K. Westerbeek. 1998. "Educational Stratification in Italy: Reforms, Expansion and Equality of Opportunity". European Sociological Review 14: 33-47.

UNESCO (varios años). Anuario Estadístico. París: UNESCO.

Unu-Wider. 2005. World Income Inequality Database, Version 2.0a, descargado de la página web http://www. wider.unu.edu/wiid/wiid.htm.

Vallet, L.A. 2004. "Change in Intergenerational Class Mobility in France from the 1970s to the 1990s and Its Explanation: An Analysis Following the Casmin Approach". En R. Breen (coord.), Social Mobility in Europe, pp. 115-48. Oxford: Oxford University Press. 


\section{Apéndice}

Tabla 3.

Estadísticos descriptivos

\begin{tabular}{|l|c|c|c|c|c|}
\hline VARIABLE & MEDIA & $\begin{array}{c}\text { DESV. } \\
\text { TÍPICA }\end{array}$ & MÍNIMO & MÁXIMO & $\mathrm{N}$ \\
\hline $\begin{array}{l}\text { Gasto público } \\
\text { educación (\% PNB) }\end{array}$ & 5.119 & 1.580 & 1.1 & 9.7 & 885 \\
\hline Gasto Terciaria/Primaria (T/P) & .510 & .2803 & .027 & 2.123 & 609 \\
\hline $\begin{array}{l}\text { Gasto secundaria (\% total } \\
\text { gasto educación) }\end{array}$ & 33.698 & 11.190 & 8.5 & 56.5 & 610 \\
\hline IZQUIERDA & .528 & .499 & 0 & 1 & 823 \\
\hline GINI & 29.743 & 6.626 & 19.7 & 49.5 & 465 \\
\hline INGRESO & 11728 & 5993 & 1038 & 35144 & 1421 \\
\hline APERTURA COMERCIAL & 62.013 & 38.790 & 7.986 & 208.643 & 1303 \\
\hline POBL <14 & 26.276 & 7.132 & 14.796 & 46.479 & 1380 \\
\hline RURAL & 31.843 & 16.274 & 2.7 & 77.9 & 1573 \\
\hline LOG(POBL) & 16.197 & 1.565 & 12.078 & 19.407 & 1380 \\
\hline RÉGIMEN & .295 & .456 & 0 & 1 & 1527 \\
\hline
\end{tabular}

DULCE MANZANO obtuvo su Máster en Ciencias Sociales Avanzadas en el Instituto Juan March y es doctora en Ciencia Política por la Universidad Autónoma de Madrid. Ha sido investigadora visitante en el Departamento de Política de la Universidad de Nueva York. En la actualidad es investigadora posdoctoral en el Instituto Juan March y profesora asociada en el Departamento de Sociología y Comunicación de la Universidad de Salamanca.

LEIRE SALAZAR es Licenciada en Sociología. Obtuvo su Máster en Ciencias Sociales Avanzadas en el Instituto Juan March y es doctora en Sociología por la Universidad de Oxford. En su tesis, investigó los efectos de los cambios en la formación de hogares -y en particular en la homogamia educativa- sobre la desigualdad de la renta. En la actualidad es profesora en el Departamento de Sociología Il de la UNED.

RECIBIDO: 25/03/08

ACEPTADO: $26 / 11 / 08$

Publicado on-line: 15/06/09 